\title{
TC-1 卫星在近地磁尾观测到的持续尾向流事件
}

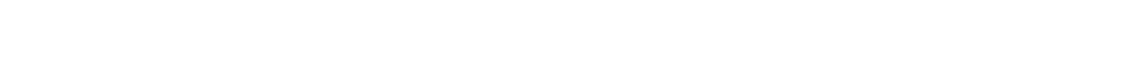 \\ (1) 中国科学院空间科学与应用研究中心, 北京 100080; (2) 中国科学院等离子体研究所, 合肥 230031; (3) 北京大学地球与空间科学 \\ 学院, 北京 100871; (4) 中央民族大学物理与电子工程学院, 北京 100081. E-mail: lqzhang@cssar.ac.cn)
}

\begin{abstract}
摘要 2004 年 7 月 11 日ACE卫星、 Imagine卫星和TC-1 卫星联合观测到伴随有持续尾向流的亚暴过程. TC-1 卫星在近地磁尾晨侧观测到的磁尾亚暴过程有三个阶段: 增长相过程(11:43 12:19), 预膨胀过程 (12:19 12:28)和偶极化过程. Imagine卫星在 12:26 观测到极光突然增亮; 2 min后偶极化过程发生. 尾向 流的持续时间约 $45 \mathrm{~min}$, 经历了增长相和预膨胀相. 随偶极化过程的发生, TC-1 卫星进入等离子体片内 观测到高速地向流. 尾向流具有明显的高密、低温和沿磁场方向流动的特征, 与Cluster等卫星在近地磁 尾观测到的电离层上行离子流特征吻合. 卫星的联合观测表明近地磁尾尾向流与南向行星际磁场密切 相关, 对亚暴过程有重要影响.
\end{abstract}

关键词 亚暴膨胀相突发 偶极化 南向行星际磁场 尾向流

磁层亚暴是地球空间最重要的能量存储和释放 过程. 由于磁层亚暴对地球空间有广泛和重要的影 响, 其全球过程、效应和模型研究一直是日地物理学 最受重视的前沿课题之一. 近 30 年来磁层亚暴研究 已经取得很大进展, 但关于膨胀相起源仍有很大争 议. 近地磁尾是亚暴膨胀相触发的主要活动区域, 粒 子注入事件、越尾电流中断、 $\mathrm{Pi} 2$ 的发生及偶极化开 始的位置都位于这个区域 $[1 \sim 4]$. 近地磁尾同时是中磁 尾和电离层活动的直接响应区域, 在电离层与磁层 耦合过程中起着核心的作用. 近地磁尾场和粒子的 研究对了解亚暴和磁暴过程的触发机制有重要的 意义 ${ }^{[5,6]}$.

上行离子作为电离层和磁尾耦合过程的重要方 式对亚暴过程有重要的影响. Cladis等人 ${ }^{[7]}$ 根据引导 中心轨迹计算得到磁层中极隙区起源 $\mathrm{O}^{+}$的粒子分布 结果, 最早提出了由于磁场和电场对上行 $\mathrm{O}^{+}$离子压 力的响应, 使上行离子有可能成为亚暴的触发机制. Daglis等人 ${ }^{[8 \sim 10]}$ 对上行 $\mathrm{H}^{+}$和 $\mathrm{O}^{+}$离子与亚暴 $\mathrm{AE}$ 指数和 亚暴增长相期间越尾电流的变化进行了观测和分析, 得到的结论如下: 伴随上行 $\mathrm{H}^{+}$和 $\mathrm{O}^{+}$离子的密度增长, 越尾电流增强, 同时 AE指数增高. Kistler等人 ${ }^{[11]}$ 进一 步使用Cluster和Image卫星对亚暴期间上行离子和极 光活动进行了多点观测, 发现上行离子与极光弧之 间有非常好的对应关系，上行离子通量最高时间与 极光弧亮度最高时间相对应. 最近的观测和模拟结
果进一步表明极区电离层上行离子对近地磁尾动力 学过程有重要的影响. 2005 年Liemohn等人 [12]利用 Polar卫星对近地磁尾(约 $9.5 \mathrm{Re}$ )的低能、冷等离子体 上行离子细流进行了统计研究. 观测结果显示离子 细流是连续的出现在尾瓣区, 形成尾瓣风. 由于上行 离子细流可以将电离层起源的离子连续和直接的提 供给近地磁尾内磁层提供电离层起源的粒子成分, 在地磁扰动时不需要通过磁层对流将远磁尾的上行 离子携带至近地. Nose等人 ${ }^{[13]}$ 和Moore等人 ${ }^{[14]}$ 研究 了南向行星际磁场驱动下向阳面和夜侧极光椭圆带 区域的上行离子, 结果表明起源于夜侧极光椭圆带 区域上行离子能够形成极光区风, 对近地磁尾(10 Re 附近) 粒等离子体片成分和等离子体片 有重要的影响.

2002 年 12 月 30 日发射的 TC- 1 卫星是近地赤道 卫星, 轨道高度 $6900 \times 85300 \mathrm{~km}$, 倾角 $28.5^{\circ}$, 开普勒 周期约 27.4 h. TC-1 卫星上配置有和磁通门磁强计 (FGM)和热离子分析仪(HIA). FGM 可以精确进行高 时间分辨的磁场三维测量, 测量范围为-65536 $+65504 \mathrm{nT}$, 最高为 $7.813 \times 10^{-3} \mathrm{nT}$, 分辨率为 $4 \mathrm{~s}$. HIA 能够测量离子的能谱、三维分布函数, 探测的能量范

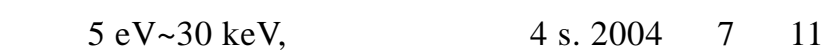
日 TC-1 卫星观测到 1 次典型的伴随有持续尾向流的 亚暴过程, 尾向流的持续时间约 $45 \mathrm{~min}$. 本文的主要 目的是通过 TC-1 卫星在 2004 年 7 月 11 日观测到的 
持续尾向流事件, 了解近地磁尾尾向流对亚暴过程 的影响.

\section{4 年 7 月 11 日亚暴事件分析}

1.1 地面、Imagine 卫星和 ACE 卫星的联合观测

（i ）亚暴 AE 指数：极光电集流的强度通常用极 光电集流指数 $\mathrm{AL}, \mathrm{AU}, \mathrm{AE}$ 和 $\mathrm{AO}$ 来描述. 这些指数 的数值由均匀分布在极光带附近各个地磁台的每个 小时内水平强度变化来决定(应消除平均的平静变 化), 水平强度的单位为纳特. $\mathrm{AU}$ 指数是在这些台站 中每个小时内的最大正变化, $\mathrm{AL}$ 指数是在这些台站 中每个小时内的最大负变化, $\mathrm{AE}$ 指数是每个小时内 最大正变化同最大负变化的绝对值之和. AE 指数是 目前普遍用来描述亚暴强度的指数. 当天 $\mathrm{AE}$ 指数的 变化如图 1 所示: 在 12:30 左右, AE 指数最高超过 500 nT.

(ii ) DST 指数: 是描述磁暴时变化的指数. 在地 磁赤道附近选取 5 个均匀分布在不同经度上的地磁 台, 这些台站的每个小时内水平强度变化的平均值 就是 DST 的数值, 单位为 nT. 当天 9:00 DST 指数达 到最高, 为 $23 \mathrm{nT}$; 由 12:00 13:00, DST 指数由 $2 \mathrm{nT}$ 变化为 $-5 \mathrm{nT}$, 没有磁暴发生.

(iii) 极光活动: Imagine 卫星于 12:26:05 观测到 了明显的极光增亮, 由此确定亚暴膨胀相触发的时 间为 12:26:05.

(iv) 太阳风和行星际磁场条件: 如图 2 所示, ACE 卫星的观测结果显示自 10:00 11:30 行星际磁场
持续南向, 在-5 nT 逐渐升高到-9 nT; 在 11:33 行星 际磁场突然南转北. 太阳风流速由 $390 \mathrm{~km} / \mathrm{s}$ 持续增 加至 $450 \mathrm{~km} / \mathrm{s}$.

\subsection{TC-1 卫星的观测结果}

2004 年 7 月 11 日 TC-1 卫星在近地磁尾观测到 的亚暴过程中磁场和流场随时间的演化如图 3 所示, 图 3 中从上到下依次为流场 $\left(V_{\mathrm{x}}\right)$ 、磁场 $\left(B_{\mathrm{x}}, B_{\mathrm{y}}, B_{\mathrm{z}}\right)$ 、密 度 $(n)$ 、温度(包括热离子温度 $T$, 平行磁力线温度 $T_{\mathrm{par}}$ 和垂直磁力线的温度 $T_{\mathrm{per}}$ ) 和流场投影(包括平行磁力 线速度 $V_{\mathrm{par}}$ 和垂直磁力线速度 $V_{\mathrm{per}}$ ).

\subsection{1 磁场特性}

( i ) 11:43 12:19 为亚暴增长相过程: 磁场 $B_{\mathrm{x}}$ 分量从 $26 \mathrm{nT}$ 逐渐升高至 $56 \mathrm{nT}$; 磁场 $B_{\mathrm{y}}$ 分量在 $30 \mathrm{nT}$ 逐渐下降至 $20 \mathrm{nT}$; 磁场 $B_{\mathrm{z}}$ 分量由 $10 \mathrm{nT}$ 逐渐下降至 $2 \mathrm{nT}$. 该过程持续时间约 $36 \mathrm{~min}$, 具有明显的亚暴增 长相特征.

(ii ) 12:19 12:28 为预膨胀过程: 磁场 $B_{\mathrm{x}}$ 分量由 $56 \mathrm{nT}$ 逐渐下降至 $53 \mathrm{nT}$; 磁场 $B_{\mathrm{y}}$ 分量从 $20 \mathrm{nT}$ 逐渐 下降至 $18 \mathrm{nT}$; 磁场 $B_{\mathrm{z}}$ 分量从 $2 \mathrm{nT}$ 迅速升高至 $12 \mathrm{nT}$. 在增长相后期磁场位型出现了与膨胀相期间相似的 变化特征, 由非偶极型逐渐向偶极型转化, 等离子体 片有较弱膨胀. 将这种在亚暴增长相后期、膨胀相触 发之前的缓慢等离子片膨胀过程称为预膨胀过程, 此过程伴随着磁场的明显扰动. 增长相期间储存在 近地磁尾的能量有可能首先在预膨胀过程中被缓慢 释放, 然后在偶极化过程中被突然释放. 与磁通量堆
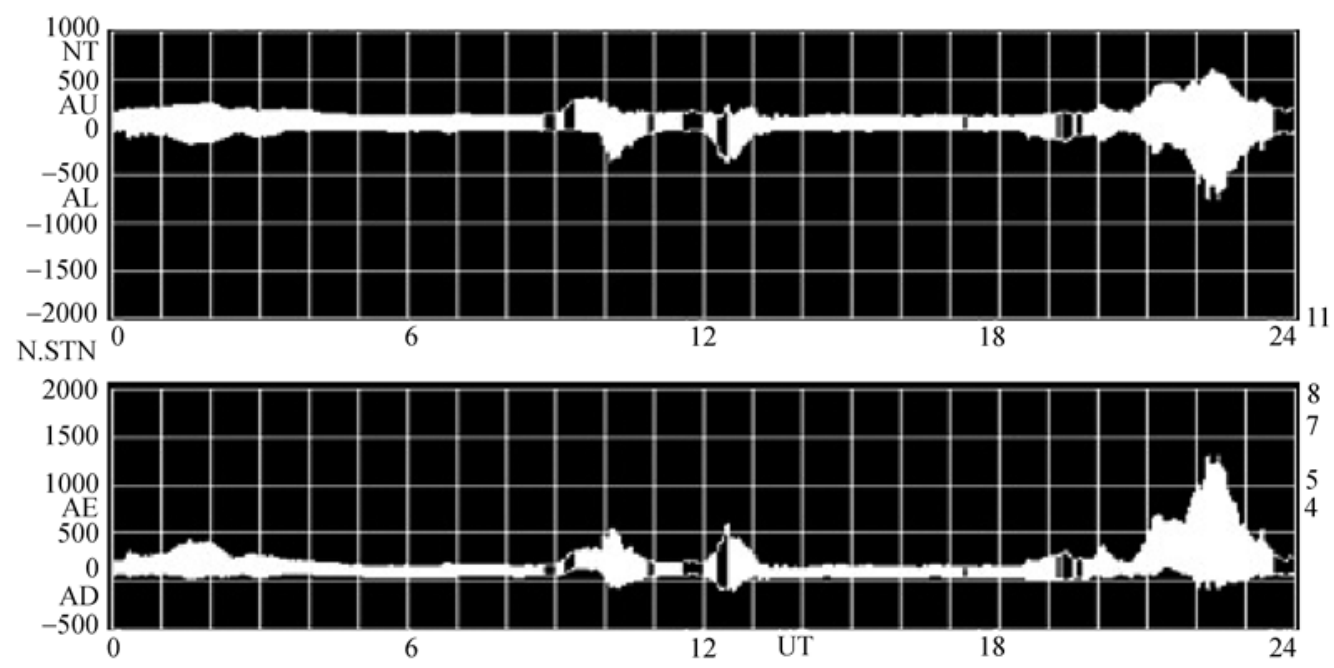

图 12004 年 7 月 11 日 AE 指数

在 $12: 30$ 左右, $\mathrm{AE}$ 指数最高超过 $500 \mathrm{nT}$, 有亚暴发生 

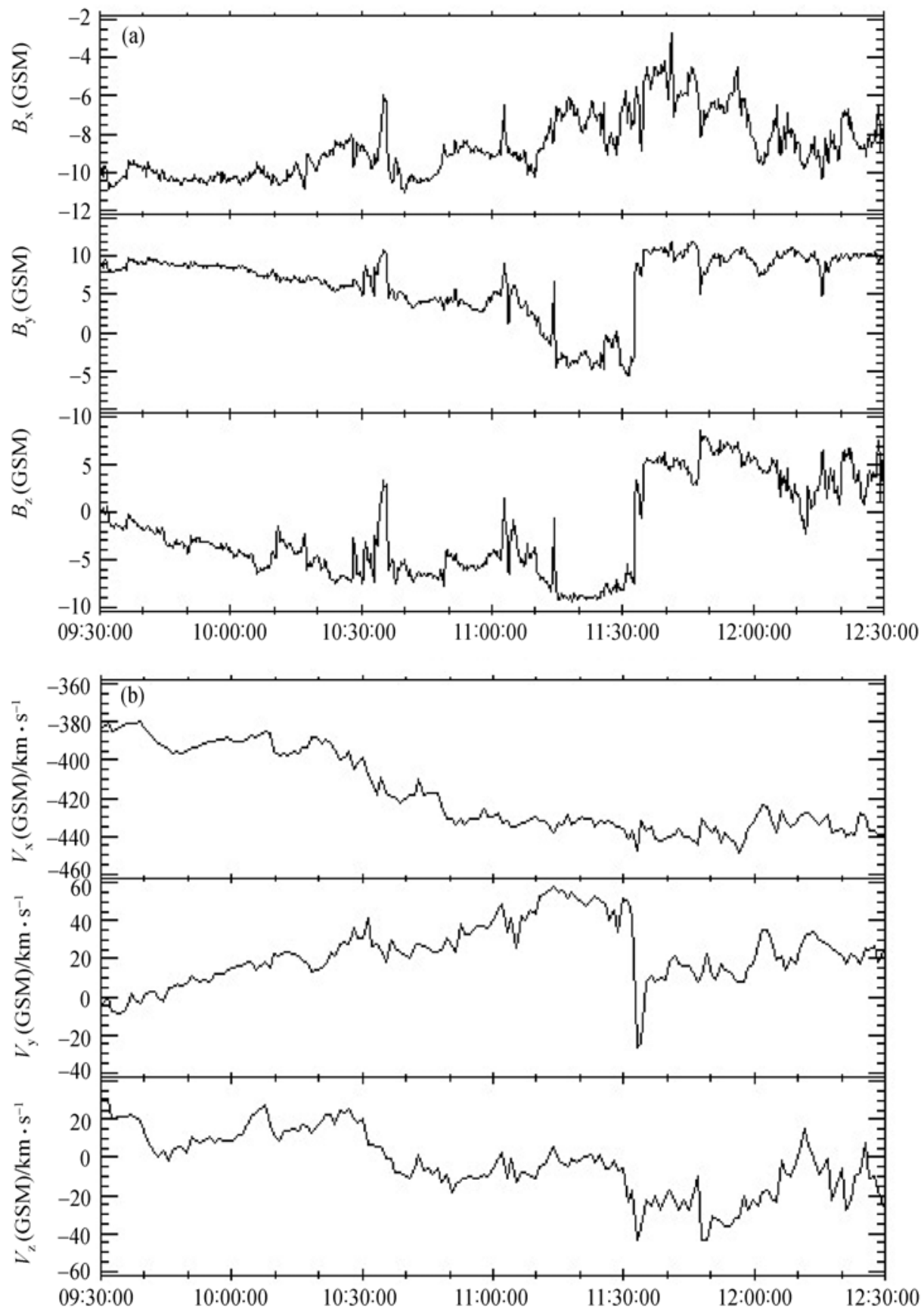

图 2 太阳风和行星际磁场条件

2004 年 7 月 11 日 10:00 11:30 期间太阳风流速由 $390 \mathrm{~km} / \mathrm{s}$ 持续增加至 $450 \mathrm{~km} / \mathrm{s}$; 行星际磁场持续南向, 在 $-5 \mathrm{nT}$ 逐渐升高到-9 nT. 在 11:33 行星际磁场突然南转北

积过程不同, 预膨胀过程中的磁场 $B_{\mathrm{x}}$ 下降, $B_{\mathrm{z}}$ 分量增 高. 与偶极化过程也不同, 偶极化过程持续时间较短 (约 2 3 min), 且磁场 $B_{\mathrm{y}}$ 分量会有突然增强. 预膨胀 过程的持续时间较长(约 $9 \mathrm{~min}$ ), 而且磁场 $B_{\mathrm{y}}$ 分量没 有较明显的变化. (iii ) 12:28 12:31 为偶极化过程: 磁场偶极化过 程持续了约 $3 \mathrm{~min}$. 磁场 $B_{\mathrm{x}}$ 分量由 $52 \mathrm{nT}$ 迅速下降至 $18 \mathrm{nT}$; 磁场 $B_{\mathrm{y}}$ 分量从 $16 \mathrm{nT}$ 突然升高至 $37 \mathrm{nT}$; 磁场 $B_{\mathrm{z}}$ 分量从 $14 \mathrm{nT}$ 迅速升高至 $25 \mathrm{nT}$. 12:31之后恢复相开 始, 和增长相开始时刻(11:43)的磁场(26, 23, $10 \mathrm{nT})$ 


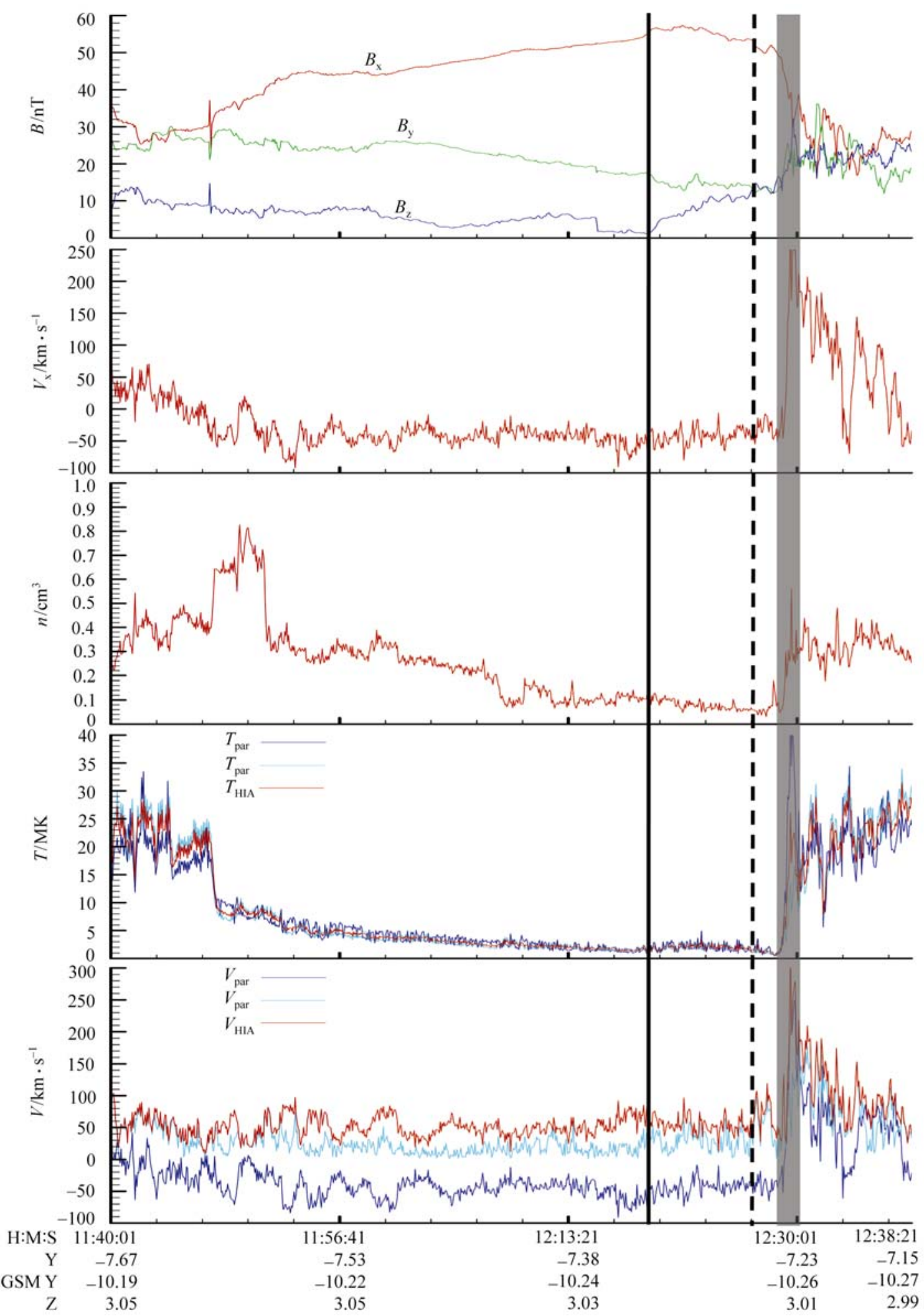

图 32004 年 7 月 11 日 TC-1 卫星在近地磁尾观测到的尾向流和亚暴过程

实线标志为预膨胀过程开始时间(12:19); 虚线标志为极光突然增亮, 亚暴膨胀相触发时间(12:26); 阴影部分为偶极化过程, 持续时间约 3 min. TC-1 卫星在近地磁尾观测到持续时间约 $45 \mathrm{~min}$ 的尾向流事件, 尾向流具有明显的高密、低温和沿磁场方向流动特征. 尾向流经历了整个增长相 和预膨胀过程. 随偶极化过程发生, 在等离子体片内观测到高速地向流 
相比, 到 12:36 磁场各个分量为( $30,14,20 \mathrm{nT})$, 磁力 线基本恢复原状.

\subsection{2 热离子特性分析}

(1) 热离子密度和温度

增长相期间尾向流速 $\left(V_{\mathrm{x}}\right)$ 最高达到 $-90 \mathrm{~km} / \mathrm{s}$, 平 均为 $-43 \mathrm{~km} / \mathrm{s} ; V_{\mathrm{y}}$ 平均为 $-13 \mathrm{~km} / \mathrm{s} ; V_{\mathrm{z}}$ 平均为 $-7 \mathrm{~km} / \mathrm{s}$. 热离子密度由 $0.8 \mathrm{~cm}^{-3}$ 逐渐下降至 $0.1 \mathrm{~cm}^{-3}$. 热离子 的温度由 $27 \mathrm{MK}$ 持续下降至 $3 \mathrm{MK}$, 平行温度 $\left(T_{\mathrm{par}}\right)$ 和垂直温度 $\left(T_{\mathrm{per}}\right)$ 同时下降, 且 $T_{\mathrm{par}}$ 和 $T_{\mathrm{per}}$ 逐渐趋向一 致. 尾向流的平行温度平均 $3.2 \mathrm{MK}$, 垂直平均温度 $3.0 \mathrm{MK}$, 热离子由各向异性逐渐趋向各向同性. 增长 相期间 TC-1 卫星的位置基本没有变化, 由(-7.5 Re, $-10.2 \mathrm{Re}, 3.0 \mathrm{Re})$ 运动至( $-7.4 \mathrm{Re},-10.2 \mathrm{Re}, 3.0 \mathrm{Re})$. 热离子密度的变化是由于增长相期间等离子体片变 薄, 卫星由等离子体片逐渐进入等离子体片边界层 造成的.

在预膨胀过程中, 热离子密度由 $0.1 \mathrm{~cm}^{-3}$ 进一步 下降至 $0.04 \mathrm{~cm}^{-3}$. TC- 1 卫星位于等离子体片边界层 附近, 并逐渐进入低纬瓣区. 预膨胀过程中尾向流热 离子温度有逐渐增高的趋势, 同时热离子的温度、

$T_{\mathrm{par}}$ 和 $T_{\mathrm{per}}$ 表现出明显的扰动.

随偶极化过程的发生, $V_{\mathrm{x}}$ 由 $-40 \mathrm{~km} / \mathrm{s}$ 突然升高至 $250 \mathrm{~km} / \mathrm{s}$, 同时热离子密度迅速升高至 $0.3 \mathrm{~cm}^{-3}$ 左右. 热离子的温度由 $1 \mathrm{MK}$ 迅速升高至 $24 \mathrm{MK}$. 同时温度 由各向同性转变为明显的各向异性. 密度的变化表 明随偶极化过程的发生, TC-1 卫星由片边界层进入 了中心等离子体片观测到高速地向流. 速度和温度 的突然变化不是尾向流结束, 而是由于 TC-1 卫星由 尾向流区域(低纬瓣区和等离子体片边界层)进入了 地向流区域(等离子体片内)造成的.

与偶极化过程出现的地向流温度(约 $25 \mathrm{MK}$ )相 比, 尾向流具有明显的低温特性. 相对磁场而言, 地 向流热离子的垂直温度高于平行温度, 尾向流的平 行温度高于垂直温度. 温度的变化表明了流场与磁 场之间可能存在着能量转化过程. 首先离子的热能 能够转化为磁能储存在近地磁尾; 在预膨胀过程中, 随磁场位型逐渐由非偶极型向非极型转化, 部分磁 能可能会转化为离子的热能.

（2）流场和磁场的夹角

尾向流的持续时间为 11:43 12:28, 约 $45 \mathrm{~min}$,
经历了整个增长相过程和预膨胀过程. 将流场在磁 场方向上投影, 可知整个尾向流持续期间尾向流和 磁场之间的夹角平均为 $104.4^{\circ}$, 尾向流基本沿磁力线 方向流动, 即尾向流具有明显的场向特征. 尾向流平 行磁场速度在 $40 \mathrm{~km} / \mathrm{s}$ 左右, 垂直磁场速度在 $10 \mathrm{~km} / \mathrm{s}$ 左右, 平行磁场速度明显较垂直磁场速度高.

\section{2 讨论}

目前对近地磁尾尾向流的起源一般认为有两种: (i) 直接起源于电离层的极光椭圆区域, 这是被 ISEE-1 卫星和Polar卫星证实的. 这部分尾向流的主 要特征是低温、高密, 主要分布在低纬斑区, 片边界 层和等离子体片内; ( ii ) 被电离层反射回来向磁尾 方向流动的离子束, 主要分布在等离子体片边界层, 且能量较高. 本事件中观测到的连续尾向流具有电 离层上行离子流的明显特征, 如低温、高密和沿磁力 线流动等特征, 这与ISEE-1 卫星和Polar卫星在近地 观测到来自电离层的尾向流特征基本吻合, 且卫星 在等离子体片边界层和低纬瓣区等区域连续观测到 了尾向流, 表明其来自电离层. Cluster卫星在近地磁 尾(4 Re附近)曾观测到类似的持续时间超过 $30 \mathrm{~min}$ 上 行离子流事件 ${ }^{[11]}$. 上行离子的流速平均在 $50 \mathrm{~km} / \mathrm{s}$ 左 右, 上行离子的 $\mathrm{O}^{+}$数密度较 $\mathrm{H}^{+}$的高. 这一事件中, Cluster卫星位置处的磁力线与夜侧极光椭圆带相连, 上行离子通量与极光弧亮度最高相对应. 这一观测 表明起源于夜侧极光椭圆带区域的上行粒子和亚暴 过程有密切的关系.

TC-1 卫星在近地磁尾, 包括晨昏两侧和夜侧的 低纬瓣区、等离子体片边界层和等离子体片区域都观 测到大量的尾向流事件. 对这些尾向流事件的统计 分析表明近地磁尾尾向流具有明显来自电离层的高 密、低温特征 ${ }^{[15]}$. 由此可以知道 TC-1 卫星在近地磁 尾观测到的尾向流应该是来自电离层, 对近地磁尾 动力学过程有重要的影响 ${ }^{1)}$. 本文对了解近地磁尾尾 向流对亚暴过程的影响提供了观测依据.

\section{3 结论}

2004 年 7 月 11 日 ACE 卫星、 Imagine 卫星和 TC-1 卫星联合观测到行星际磁场南转北触发的亚暴 过程. ACE 卫星观测到 10:00 11:30 期间行星际磁场 持续南向, 在 11:33 行星际磁场突然南转北; 自 11:43

1) Zhang L Q, Liu Z X, Pu Z Y, et al. The effect of the tailward flow original from the ionosphere on the near Earth magnetic field. Chin J Space Sci, 2007, 27(3). 已接受 
开始, TC-1 卫星在近地磁尾晨侧( $-7 \mathrm{R}_{\mathrm{e}},-10 \mathrm{R}_{\mathrm{e}}, 3 \mathrm{R}_{\mathrm{e}}$ ) 观测到亚暴增长相过程开始, 增长相持续时间约 36 $\min$; 在增长相后期(12:19 起)有预膨胀过程发生, 持 续时间 9 min; Imagine卫星在 12:26 观测到极光突然 增亮，亚暴膨胀相触发; 2 min后偶极化过程发生,持 续时间约 $3 \mathrm{~min}$. 亚暴过程中伴随有持续约 $45 \mathrm{~min}$ 尾 向流，经历了整个亚暴增长相过程和预膨胀过程. 尾 向流分布在等离子体片边界层和低纬瓣区，具有明 显的高密、低温和沿磁场方向流动特征. 等离子体片 内来自中磁尾的高温地向流与等离子体片边界层来 自电离层的低温尾向流相互作用较易激发不稳定性, 对亚暴膨胀相的触发可能有重要的影响. 本文的观 测同时表明近地磁尾尾向流与南向行星际磁场密切 相关, 这与Nose等人 ${ }^{[13]}$ 和Moore等人 ${ }^{[14]}$ 的研究结果 相吻合.

\section{致谢 感谢中国双星数据中心为本文提供所需数据.}

\section{参考文献}

1 Lui A T Y, Chang C L, Mankofsky A, et al. A cross-field current instability for substorm expansions. J Geophys Res, 1991, 96: 11389-11401

2 Shiokawa K, Baumjohann W, Haerendel G. Braking of high-speed flows in the near-Earth tail. Geophys Res Lett, 1997, 24: 11791182[DOI]

3 Yau A W, Shelley E G, Peterson W K, et al. Energetic auroral and polar ion outflow at ED 1 altitudes: magnitude, composition and magnetic activity dependence and long term variations. J Geophys Res, 1985, 90: 8417-8432

4 Lui A T Y. Current disruption in the Earth's magnetosphere: observations and models. J Geophys Res, 1996, 101: 1306713088[DOI]
5 Shelley E G, Jonson R G, sharp R D. Satellite observations of energetic heavy ions during a geomagnetic storm. J Geophys Res, 1972, 77: 6104-6110

6 Moore T E. Origining of magnetospheric plasma. Rev Geophys, 1991, (Suppl 1): 1039-1048

7 Cladis J B, Francis W E. The polar ionosphere as a source of the storm time ring current. J Geophys Res, 1985, 90: 3465—3473

8 Daglis I A, Paschalidis N P, Sarris E T, et al. Statistical features of the substorm expansion-phase as observed by AMPTE/CCE in Magnetospheric substorms. In: Kan J R, Potemra T A, Kokubun S, et al, eds. Geophysical Monography Series, 64. Washington: American Geophysical Union, 1991. 323-332

9 Daglis I A, Sarris E T, Kremser G. Ionospheric contribution to the cross-tail current during the substorm growth phase. J Atmos Terr Phys, 1991, 53: 1091-1098[DOI]

10 Daglis I A, Sarris E T, Wilken. AMPET/CCE CHEM observations of the ion population at geosynchronous altitudes. Ann Geophys, 1993, 11: 685-696

11 Kistler L M, Frey H U, Mobius E, et al. Motion of auroral ion outflow structures observed with Cluster and IMAGE FUV. J Geophys Res, 2002, 107: 1186-1192[DOI]

12 Liemohn M W, Moore T E, Craven P D, et al. Occurrence statistics of cold, streaming ions in the near-earth magnetotail: Survey of Polar-Tide observation. J Geophys Res, 2005, 110: 2687-2704

13 Nose M, Taguchi S, Hosokawa K, et al. Overwhelming $\mathrm{O}^{+}$Contribution to the plasma sheet energy density during the October 2003 superstorm: Geotail/EPIC and IMAGE/LENA observations. J Geophys Res, 2005, 110: doi: 10.1029/2004JA010930

14 Moore T E, Fok M C, Christon S P, et al. Solar and ionospheric plasmas in the ring current region. Geophys Monogr, 2005, 159: 179-194

15 张灵倩, 刘振兴, 王继业. 近地磁尾流场的分布特性. 科学通报, 2007, 52 